\title{
Vitamin D and Biphosphonate in Neoadjuvant Advanced Breast Cancer
}

\author{
Selma Sakhri* \\ Medical oncology, Algeria \\ *Corresponding author: Selma Sakhri, Medical oncology, Algeria \\ Submission: 監April 09, 2018; Published: 海June 19, 2018
}

\begin{abstract}
Vitamin D and calcium deficiency is associated with increased breast cancer risk and decreased breast cancer survival. The purpose of this study is to determine whether the addition of vit $\mathrm{D}$ and Zoledronic acid (ZA) to neoadjuvant chemotherapy (NACT) gives complete histological responses. We report a prospective evaluation comparing complete pathological response between different biomolecular sub-groups.
\end{abstract}

Patients and Methods: The study included 431 women with stages III locally advanced breast cancer who received neoadjuvant chemotherapy and Zoledronic acid. All patients were prescribed vitamin D3 (cholecalciferol) $400 \mathrm{IU}$ and calcium carbonate 1,000 mg daily. The main objective is the complete histologic response. Secondary end points were the over all survival of patients targeted by the study.

Results: Histologic complete response with Zoledronic acid and vit D was $40.13 \%$ the higher in the subgroup Her 2/luminal (RH \pm Her2+) and under Her2+ (HR-Her2+) and the lowest rate was observed in the triple negative group as classified by Sataloff, overall survival was 45.77 months for subgroups (Her2 / luminal and in Her2 + group) vs 44.11 months for triple negative group.

Conclusion: Our study confirms the potential protective and antiproliferatif effects with vitamin D and Zoledronic acid in neoadjuvant advanced breast cancer.

Keywords: Zoledronique acid; Breast cancer; Neoadjuvant chemotherapy; Vitamin D; Calcium

\section{Introduction}

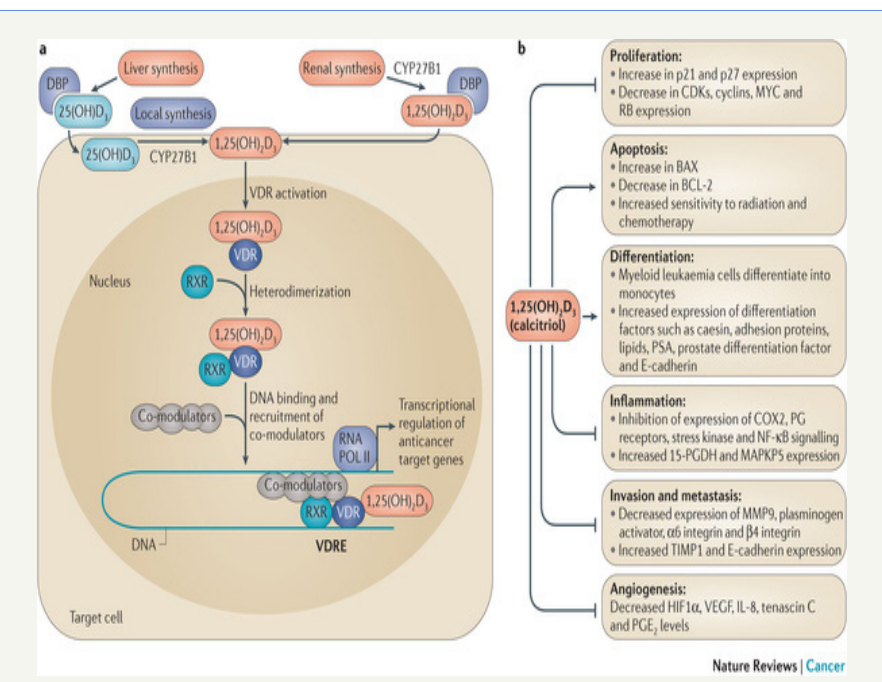

Figure 1: Antitumor effects of vitamin D.

Preclinical studies support various antitumor effects of vitamin D and Zoledronic acid (Neoazure trial) in breast cancer (BC) such as inhibition of cell proliferation, induction of cell differentiation, promotion of apoptosis, decreasing inflammation with down regulation of cyclooxygenase-2, decreasing of angiogenesis, inhibition of estrogen pathway and also inhibition of invasion and metastasis $[1,2]$. Due to the increasing concern of the role of vitamin D in the development and prognosis of the $\mathrm{BC}$, also it enhances chemotherapy induced cell death by increasing the pathologic complete response (PCR). Pubmed search. Because of the intracellular effects on breast cancer cells and the in vitro and in vivo experiments demonstrating enhancement of chemotherapeutic cytotoxicity with vit D pretreatment, we hypothesized that low vit D levels would be associated with impaired response to chemotherapy and more aggressive breast tumor biology resulting in higher relapse rates among women with vit D deficiency (Figure 1,2).

\section{Methods}

From 2012 to 2014, 432 patients received neoadjuvant chemotherapy + Zoledronic acid and vitamin D. The primary end point was to determine the relation between vit $\mathrm{D}+$ Calcemia levels and pathologic complete response. (PCR) when adding Zoledronic acid. 
The secondary end point was the overall survival. Of all patients $(\mathrm{N}=438), 432$ received neoadjuvant chemotherapy + vitD and zoledronic acid. Six of them have progressed after chemotherapy treatments and 04 are excluded from the study, at the end of neoadjuvant chemotherapy, a patient refused surgery therefore no assessment of pCR in this patient who is the main objective of our study, a total of 7 patients study outputs, so it remains 431 who have had a mastectomy with lymph node dissection seen the very advanced stage of the tumor (IIIA, IIIB, IIIC) and the appointment of delay Involved in the period radiotherapy (7-15-month). No conservative treatment has been practiced. The median follow-up of patients was 42 months.

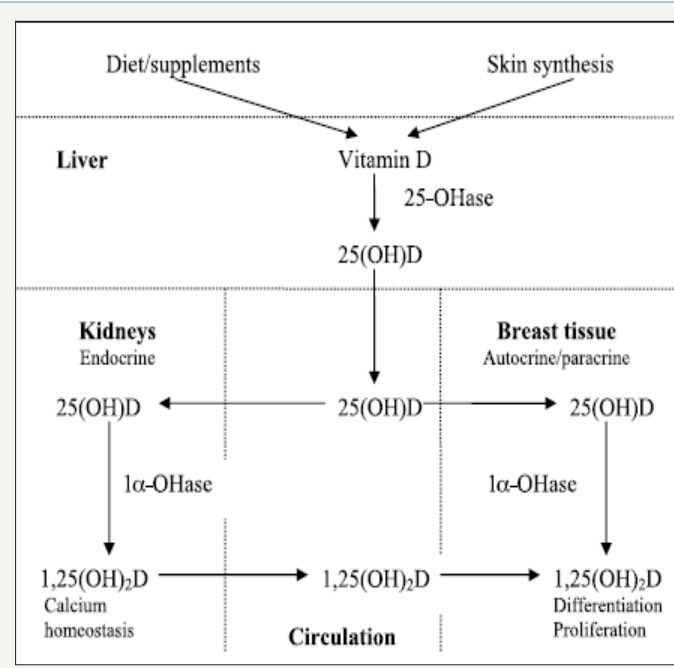

Figure 2: Diagram of vitamin D metabolism.

\section{Result}

The pathologic complete response rate was $40.13 \%$ according to classification Sataloff (1995).We found that the pathologic complete response rate was the highest in the subgroup (Her2 / luminal) and (Her2 +) and the lowest rate in the triple negative group as classified by Sataloff. These results are consistent with a single study of literature is the study Rouzier (10) where the pathological complete response to neoadjuvant chemotherapy in patients who overexpressed Her2 + is obtained even in the absence of trastuzumab. This leaves suggest that the answer is guided by specific biological factors in Her2 + tumors and zoledronic acid plus vit $\mathrm{D}$ may play a role in inhibiting tyrosine kinases with its potentiation of treatment. Thus the triple negative patients are insensitive to zoledronic acid and vit D in neoadjuvant. The average duration of progression-free survival was also significantly in the subgroup (Luminal-Her2, Her2) to the triple negative. It is 45.18 months in the group (luminal - Her2 +) vs 38.95 in the triple negative group. As against the overall survival has improved in the 4 groups with 45.77 months for (luminal - Her2), 45.32 months for Her2 group, 44.37 months for luminal and 44.11 months for the TN group higher compared to the literature or overall survival of 30 months TN. SG month: Luminal / Her2; luminal; Her2 vs TN $=45.18$ months [ 45.15 to 46.21 ]; 45.26 months [45.03 to 46.50 ]; 44.18 months [40.06 to 45.30 ] vs 40.11 months [36.16 to 41.06] (95\%) (Figure 3,4).

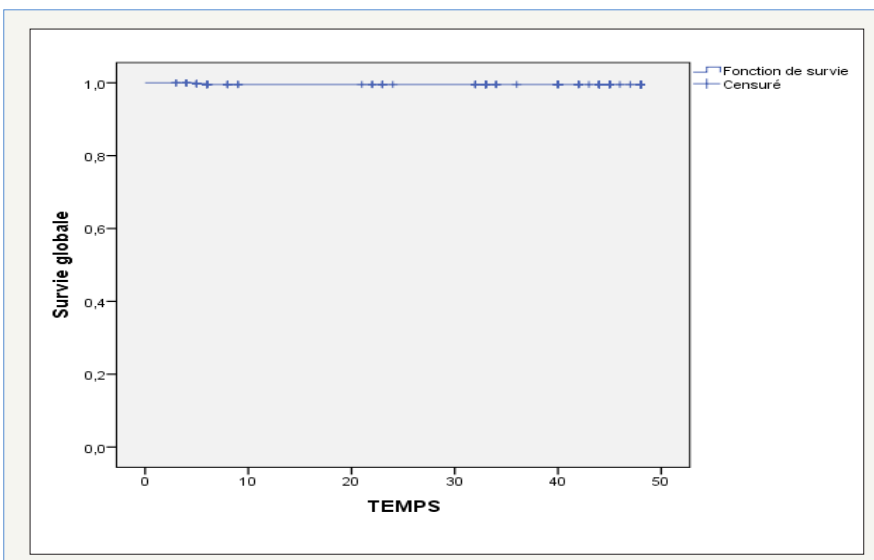

Figure 3: SG (Global Survival) Avg $=45.07$ months [43.54 to 45.81 ] (95\%).

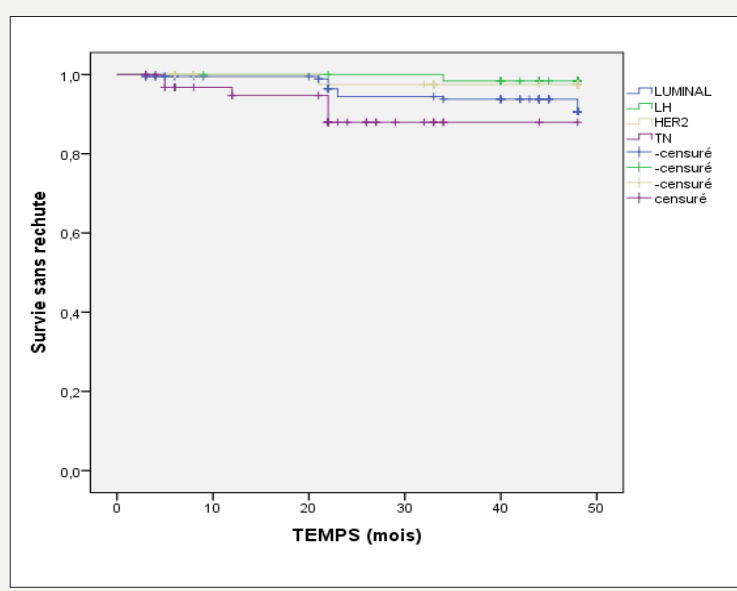

Figure 4: $\mathrm{P}=0.04$ Log Rank Test.

\section{Conclusion}

Our study suggests that vit D associated with Zoledronic acid predict the efficacy of NACT in breast cancer patients; showing synergic antitumor activity. To further confirm the potential protective effects of calcium and vitamin D on breast cancer, well-designed cohort studies and clinical trials are warranted.

\section{References}

1. Colman R (2010) The effects of zoledronic acid to neoadjuvant Adding chemotherapy on tumor response: exploratory evidence for live antitumor activity in breast cancer. Br J Cancer 102(7): 1099-1105.

2. Yan C, Thomas ER (2006) Vitamin D, calcium, and breast cancer risk: A Review. Cancer Epidemiology, Biomarkers and Prevention 15(8): 14271437. 
NACS Nover Approaches
in Cancer Study

For possible submissions Click Here
Submit Article

\section{Novel Approaches in Cancer Study}

\section{Benefits of Publishing with us}

- High-level peer review and editorial services

- Freely accessible online immediately upon publication

- Authors retain the copyright to their work

- Licensing it under a Creative Commons license

- Visibility through different online platforms 